https://helda.helsinki.fi

\title{
Light-scattering evolution from particles to regolith
}

\section{Videen, Gorden}

2015-01

\begin{abstract}
Videen , G \& Muinonen , K 2015 , ' Light-scattering evolution from particles to regolith ' , Journal of Quantitative Spectroscopy \& Radiative Transfer , vol. 150 , pp. 87-94 . https://doi.org/10.1016/j.jqsrt.2014
\end{abstract}

http://hdl.handle.net/10138/158411

https://doi.org/10.1016/j.jqsrt.2014.05.019

acceptedVersion

Downloaded from Helda, University of Helsinki institutional repository.

This is an electronic reprint of the original article.

This reprint may differ from the original in pagination and typographic detail.

Please cite the original version. 


\title{
Light-scattering evolution from particles to regolith
}

\author{
Gorden Videen ${ }^{1,2, *}$ and Karri Muinonen ${ }^{3,4}$ \\ ${ }^{1}$ Army Research Laboratory, Adelphi, MD 20783, USA \\ ${ }^{2}$ Space Science Institute, Boulder, CO 80301, USA \\ ${ }^{3}$ Department of Physics, P.O. Box 64, FI-00014 University of Helsinki, Finland \\ ${ }^{4}$ Finnish Geodetic Institute, Geodeetinrinne 2, P.O. Box 15, FI-02431 Masala, \\ Finland \\ *telephone: +1 301 394-1871, e-mail: gorden.w.videen.civ@mail.mil
}

\begin{abstract}
The radiative-transfer coherent-backscattering (RT-CB) model is unique among lightscattering methodologies as it can be used to calculate accurate light-scattering properties of sparsely populated particle volumes with sizes ranging from subwavelength to infinity. We use the RT-CB model to examine the evolution of light-scattering properties as a volume of particles increases from wavelength-sized to several hundreds of wavelengths. We examine the evolution of light-scattering intensity phase function and polarization, as well as linear and circular polarization ratios. We confirm the expected trends for backscattering features to shift to smaller phase angles as the volume increases. In addition, we also see the amplitude of these features increases to some maximum for volumes having size parameters $k R \sim 100$, before decaying to less than half this amplitude as their volumes approach infinity.
\end{abstract}

Keywords: light scattering; agglomerate; polarization; coherent backscattering; radiative transfer; opposition effect 


\section{Introduction}

Over the last few decades, significant progress has been achieved in calculating the scattering properties from irregularly shaped particles (cf., recent special issues in this journal $[1,2])$. Significant theoretical effort has resulted in different algorithms to meet this challenge. One deficiency of the methodologies is they can be applied over only a limited range of particle sizes. Numerical methods like the discrete dipole approximation (DDA) [3-5] and the finite-difference time-domain (FDTD) algorithms [6-8] require significant computational resources as particle size grows with respect to wavelength, which currently restricts the maximum practical particle size. One further exacerbation is that for irregularly shaped particles, interest often is centered on light-scattering properties averaged over particle orientation, which can increase the number of calculations by orders of magnitude. Various $T$ matrix algorithms are more efficient at calculating such average light-scattering properties $[9,10]$, but ultimately require performing matrix inversions that can be unstable as the system size grows. At the other end of the range of size parameter, ray-tracing algorithms are efficient at calculating light-scattering from particles that are large compared to the wavelength. However, the ray-tracing approximations are only valid for large, smooth bodies and tend to fail when particles size approaches the wavelength of the incident light. It is the intermediate range of particle size that has yet to be explored, although the gap between the large and small particles continues to decrease.

Interest in this intermediary region between large and small particles is also application driven. Improved algorithms and computational abilities have made it possible to calculate light-scattering properties from highly irregular particles that resemble dust or agglomerated debris $[11,12]$. The light scattering from such model particles has been shown to agree well with that of real dust particles [13]. Clusters of spheres are another irregularly shaped model particle for which the scattering properties can be calculated exactly using the superposition $T$-matrix method (STMM) [14,15]. The scattering features of these particles have been shown to resemble those of naturally occurring particles $[16,17]$. This resemblance has been used to retrieve physical properties associated with atmospheric aerosols [17,18] and cometary dust [19-28], for instance. For these reasons different particle types have been generated and their properties studied ([26-30], for instance).

One issue that has arisen relates to the behavior of the scattering phase function as the sizes of these irregular particles grow into the intermediate range. For example, the light scattered by cometary coma tends to be polarized in the TM state at small phase angles $\alpha<$ 
$20^{\circ}$. Because polarization usually is defined as the difference between the intensities of the TE state and TM state, the polarization acquires a negative sign and this region is referred to as the negative polarization branch. It has been observed that the light scattered by the innermost region of the coma may have a very strong negative polarization, with depths reaching $P_{\min } \sim-6 \%$ [31]. This region has been referred to as the circumnucleus halo and can present modeling challenges. While both the agglomerate debris particles and particles composed of clusters of spheres can achieve a deep negative polarization branch, the clusters of spheres cannot achieve this depth when the scattering properties are integrated over size distributions of particles that have been measured previously in cometary coma. In order to achieve such levels of polarization, other particle shapes are incorporated within the distributions [24, 32]. This necessarily results in additional fitting parameters, which is undesirable. However, due to current calculation limitations, the effect of larger particles on the polarization is unknown and it is possible that such a polarization may be achieved if the light scattered from these particles is especially polarized in the TM state. The question arises as to how the phase function evolves as the particle size increases. In simulations of the lightscattering properties of agglomerated debris particles, a large negative polarization branch is shown to have a maximum for wavelength-sized particles. As the particle size further increases, the amplitude of the negative polarization tends to decrease [33]. Within this decay may be smaller, higher-order maxima associated with the interference dial mechanism outlined by Muinonen et al. (2011) [34]. However, these features depend on morphology and complex refractive index [33], and may be quite different for sparse agglomerates of small spheres. Answering the question of how the polarization of a sparse cluster of spheres evolves as the cluster size increases is one of the motivations of this work.

While one approach to calculating optical properties of particles in the intermediate region has been to improve calculation efficiency in numerically intense algorithms like the DDA and the FDTD, another approach is to increase the range of applicability of ray-tracing type codes into this regime. A recent approach is to improve radiative-transfer (RT) codes by incorporating the physical mechanisms that are important when sizes and distances decrease to the order of the wavelength. One of these mechanisms is coherent backscattering (CB) that occurs when rays traveling reciprocal paths interfere with each other. It is known that this mechanism is responsible for the enhanced backscatter peak and associated negative polarization [35]. The effects of the near-field on the interaction terms have not been incorporated into the RT-CB, and it is necessary to consider sparse agglomerates until the 
effects are known. Some investigation along this line has been performed by Petrova, Tishkovets, and Jockers (2007) [36].

\section{Method}

The particles under consideration in this study are fluffy agglomerates composed of random particulate media contained within a spherical volume of radius $R$ that fully encloses $N$ identical, non-overlapping spheres of radius $r$ and refractive index $m$. The packing density of the resulting medium is $v=N r^{3} / R^{3}$. The wave number and wavelength of the incident light are $k$ and $\lambda$, respectively. The positions of the individual spherical particles are chosen to be random and uniform within the nonabsorbing volume.

The RT-CB method is based on a Monte Carlo integration of the contributions of separate rays scattered into the far-field from the volume of particles [37]. The rays trace ladder and cyclical paths and CB is incorporated using the reciprocity relation [38]. The method has been generalized to include phenomenological and other types of particles [39]. Most significantly, results of the RT-CB method have been compared with exact results calculated using the superposition $T$-matrix method (STMM) for finite volumes of spherical particles by Muinonen et al. (2012) [40]. The results demonstrate strong quantitative agreement, which verifies the technique. Controlled laboratory experiments also have been designed to measure the Stokes parameters to quantify the stringency of the approximations [41]. Descriptions of the RT-CB are given in Muinonen and Videen (2012) [39] and Muinonen et al. (2012) [40]. It should be noted that the RT-CB method is based on two requirements: $v<<1$ and $N>>1$ [39]. The first condition ensures that each particle is located in the far-field zones of all the other particles. The second condition greatly reduces the probability that a ray path will include the same particle more than one time. No simulations have yet been made on the stringency of these requirements, and that task is reserved for a future endeavor.

The goal of this work is to examine how light-scattering properties change as a function of particle size through the intermediate range between small and large particles. We use the RT$\mathrm{CB}$ method to calculate the properties of agglomerates of spherical particles as a function of the overall spherical cluster size $k R$. We begin with a cluster that is approximately the size of the illuminating wavelength $\lambda$. We then increase it in steps by three orders of magnitude. We expect the optical properties of the largest agglomerate particles to approach those of semiinfinite media. 
In our simulations, we consider four observable parameters, the total intensity phase function that is proportional to the Mueller matrix element $P_{11}$, the degree of linear polarization $P=-P_{21} / P_{11}$, the linear polarization ratio,

$$
\mu_{L}=\frac{P_{11}-P_{22}}{P_{11}+2 P_{21}+P_{22}}
$$

and the circular polarization ratio

$$
\mu_{C}=\frac{P_{11}+P_{44}}{P_{11}-P_{44}} .
$$

These represent four of the most commonly measured optical observables used in remote sensing.

\section{Results and Discussion}

RT-CB computations are carried out for specific random agglomerates composed of spherical monomers of refractive index $m=1.50$ and size parameter $k r=1.76$. We consider two different packing densities of $v=3 \%$ and $6 \%$. The Monte Carlo RT-CB simulations begin with 100,000 incident rays and require several processor hours for each case. Larger agglomerate systems require more rays than smaller agglomerates because of the higher probability of additional interactions due to the larger number of spheres composing the agglomerate.

Figure 1 shows the optical properties of four different-sized agglomerates, organized in four rows and two columns. The first column is for agglomerates having packing densities $v=$ $3 \%$ and the second is for agglomerates having packing densities of $v=6 \%$. The separate rows show, from top to bottom, the intensity phase function, linear polarization, linear polarization ratio, and circular polarization ratio. Each panel shows the phase-angle dependence for agglomerates having sizes $k R=10,30,100,300,1000,3000$. The $k R=3000$ case is in bold, but the sizes also can be distinguished through their backscattering response: as the particles become larger, the backscattering features move to smaller phase angles. Figure 2 is identical to Figure 1 except that the range of phase angles spans from $0^{\circ}$ to $30^{\circ}$ in the backscattering direction.

The first thing to note is that the forward-scattering region ( $\alpha$ approaches $180^{\circ}$ ) is dominated by diffraction effects that are not incorporated into the RT-CB model. These effects result in the forward-scattering peak. Comparisons with the STMM in Muinonen et al. (2012) [40] show the extent of the errors in neglecting this effect. For example, for a $k R=40$ agglomerate, the region from $\alpha=180^{\circ}-160^{\circ}$ are dominated by diffraction that produces a 
large peak that does not appear in these simulations. The magnitude of this peak increases, and its angular extent decreases, with agglomerate size.

\section{Intensity Phase Function}

The top panels in Figures 1 and 2 show the total intensity phase function that is normalized at phase angle $\alpha=0^{\circ}$. There are several trends that become apparent in these sets of curves. As the agglomerate increases in size, the forward scattering that is not part of the diffraction peak decreases in magnitude until it becomes smaller than the backscattering region. For agglomerate size parameters $k R \sim 300$, the phase-function has little dependence on phase angle $\alpha$, except for the forward diffraction peak and the enhancement in the backscattering region. The enhanced backscatter is more clearly shown in Figure 2.

Increasing the packing density $v$ tends to enhance the backscattering portion of the intensity phase function. This is consistent with the $\mathrm{CB}$ mechanism, since increasing the packing density reduces interparticle separations, hence, increasing particle-particle interaction. These results are consistent with measurements of compressed and uncompressed particulate substrates described by Bondarenko et al. (2006) [42] and with the numerical experiments performed by Tishovets and Petrova (2013) [30]

As the size of the agglomerate increases, the width of the backscattering enhancement decreases approximately inversely proportional to agglomerate size up to $k R \sim 100$ (see Figure 2). A transition seems to occur at this point, and further increasing the agglomerate size has a much weaker effect on the width and magnitude of the peak. By the time $k R \sim 300$, further increasing the agglomerate size has little effect on the backscattering peak height or width, and it would appear that once the agglomerate has reached this size, the backscattering intensity properties resemble those of an extended medium.

\section{Polarization}

The second row of panels of Figures 1 and 2 show the percent polarization as a function of phase angle $\alpha$. This function has a negative polarization branch at small phase angles, and a larger-amplitude positive polarization branch that peaks at $\alpha \sim 90^{\circ}$. There are several trends that become apparent in these sets of curves.

As the agglomerate size increases, the positive polarization maximum decreases in amplitude and shifts its position toward the forward-scattering region. This behavior has been seen previously in sphere clusters [25, 27, 30, for instance], as well as for Gaussian random [12] and agglomerated debris particles [11]. What has not been noted in previous studies is 
that a transition again appears in the trends near $k R \sim 100$, and that beyond $k R \sim 300$, the peak magnitude remains nearly constant at $P_{\max } \sim 12 \%$. Plots of some common light-scattering parameters as a function of agglomerate size $k R$ are shown in Figure 3. Figure $3 \mathrm{~b}$ shows the magnitude of maximum polarization decreases almost linearly with particle size $k R$ until $k R \sim$ 100 , at which point its rate of change greatly decreases. Figure $3 \mathrm{~d}$ shows the dependence of $\alpha_{\max }$ position on size $k R$. Unlike peak amplitude, the peak position continues to increase almost linearly with $k R$ well beyond $k R \sim 100$.

The negative polarization branch has significantly different behavior than the positive polarization branch. As the agglomerate size increases, there are distinct transition regions that occur at $k R \sim 100$, where a minimum amplitude is reached in the negative polarization minimum at $P_{\min } \sim-8 \%$. Interestingly, the position of this deepest minimum (Figure 3) appears to depend on packing density $v$, and occurs at smaller $k R$ for the larger packing densities. Also of interest is that for small particles, $P_{\min }$ is deeper for greater packing densities; however, after passing through the region of deepest minimum, $P_{\min }$ is deeper for lesser packing densities. As the particle approaches a semi-infinite medium, the polarizations appear similar, but it appears the asymptote for the higher-density medium is reached more rapidly. For such media, Bondarenko et al. (2006) [42] found the depth of $P_{\min }$ did increase with packing density, but that the differences increased with particle size. For $0.5 \mu \mathrm{m}$ spheres, the differences between the minima were less than $0.2 \%$, but as the sphere size increased to $1.5 \mu \mathrm{m}$, the differences were nearly $1.5 \%$. It appears that when $k R \sim 1000$, the polarization minimum is approaching an asymptotic limit of $P_{\min } \sim-2 \%$, approximately one-fourth its maximum value.

The angular position of the polarization minimum $\alpha_{\min }$ also appears to pass through a transition region at $k R \sim 100$ (Figure 3). For smaller particle sizes, the minimum position shifts to smaller phase angles as $k R$ increases. This is consistent with the CB mechanism for increasing particle separation distances and the greater pathlengths experienced by sparse agglomerates. However, once the particle grows significantly greater than the mean free pathlength, saturation is reached and $\alpha_{\min }$ now approaches the asymptotic limit of a semiinfinite medium.

Unlike the angular position of the polarization minimum $\alpha_{\min }$, the angular position of the polarization maximum $\alpha_{\max }$ does not pass through an obvious transition region at $k R \sim 100$. It continues to increase as $k R$ increases. Unlike the position of the polarization minimum, $\mathrm{CB}$ does not play a role in the position of the polarization maximum. This position is determined 
by a number of factors, including the interference of the individual rays from the different particles making up the agglomerate.

\section{Linear Polarization Ratio}

The third row of panels of Figures 1 and 2 shows the linear polarization ratios. Like the polarization phase function, the linear polarization ratios have a branch in the backscattering that depends on coherent backscattering and another branch at intermediate phase angles that does not.

The primary feature is a large-amplitude maximum that appears in the backscattering region at $\alpha \sim 50^{\circ}$. The width and height of this maximum grow with particle size $k R$, and the amplitude approaches unity as $k R$ approaches infinity. Within the backscattering region (third row of Figure 2), minima appear that are remarkably similar to those of the polarization response (second row of Figure 2). While the overall amplitude of the linear polarization ratio is greater for the denser medium, as the particle size increases $k R>>1$, the responses appear to reach the same limiting values. In Figure 3 we present the linear polarization response in the exact backscatter $\left(\alpha=0^{\circ}\right)$, which is an easily measured remote-sensing parameter. While this parameter tends to be greater for the denser particles, they approach the same value as $k R$ grows toward infinity. One trait of the linear polarization ratio is that the backscattering maximum becomes a minimum as slabs become optically thick. This was demonstrated as sphere density increases for the same size particles [43]. In Figures 2 and 3, we see this occur as particle size increase while density is held constant.

\section{Circular Polarization Ratio}

The fourth panels of Figures 1 and 2 show the circular polarization ratios. These ratios appear more complicated than the linear polarization ratios, since multiple inflection points occur for smaller particles. As particle size grows, this structure becomes smoother and the dependence on particle density disappears. Within the backscattering region (Figure 2), a sharp maximum appears as $k R$ increases. For $k R>100$, the HWHM of this peak appears to remain nearly constant. In Figure 3 we present the circular polarization response in the exact backscatter $\left(\alpha=0^{\circ}\right)$, which is another easily measured remote-sensing parameter. Like the linear polarization ratio, the circular polarization ratio tends to be greater for the denser particles, and approaches the same value, regardless of density, as $k R$ becomes grows toward infinity. 


\section{Conclusion}

We have used the RT-CB method to examine the transition of light-scattering characteristics as particle size increases from being approximately the size of the wavelength to several hundred times that of the wavelength. Many of the properties do not undergo a smooth, monotonic transition. Properties associated with the coherent backscattering mechanism, for instance, may have a maximum at an intermediary region when the particle size $k R \sim 100$, and then approach a final value asymptotically. This asymptotic value corresponds with that of an extended, semi-infinite medium.

One primary motivation for this effort was to determine the behavior of the linear polarization of scattered light from clusters of spheres as the cluster size increases. Such particles have been used for modeling cometary dust, and the inclusion of large particles, in the form of chunks of ice much larger than the wavelength, may have a significant impact on size-distributions retrievals. In any case, the shape of the negative polarization branch of large ensembles of fluffy, high-albedo objects in the solar system does resemble that of aggregate particles, and it is necessary to understand its dependence on particle size. Such pieces would be expected to have polarization signatures resembling those of an extended medium. Experimental studies have found similarities between slabs of particles and the single particles that make up these slabs [44-46]. While such a relationship may exist, the transition from single particle to extended medium is not simple. In our simulations we find that rather than a monotonically increasing polarization amplitude with increasing particle size, the amplitude of the negative polarization reaches a minimum of $P_{\min } \sim-8 \%$ at $k R \sim 100$, followed by an asymptotically decaying polarization to $P_{\min } \sim-2 \%$ for an extended medium. This suggests that the inclusion of large chunks of rock cannot account for the high level of negative polarization $\left(P_{\min } \sim-6 \%\right)$ observed in the circumnucleus halo region. It also is consistent with the dial mechanism [31] and simulations of agglomerated debris particles [24]. One significant difference between the sparse agglomerates of spheres and the agglomerated debris particles is the size dependence of the minimum. For sparse agglomerates of spheres, this minimum occurs at significantly larger sizes $(k R \sim 100)$ than for the agglomerated debris particles $(k R \sim 10)$. It is not known whether this difference is the result of the morphological or size differences of the elements making up the cluster.

Acknowledgements: Research funded, in part, by the ERC Advanced Grant No 320773 entitled "Scattering and Absorption of Electromagnetic Waves in Particulate Media" 
(SAEMPL), the Academy of Finland (contract 257966), NASA Outer Planets Research Program (contract NNX10AP93G), and NASA Lunar Advanced Science and Exploration Research Program (contract NNX11AB25G).

\section{References}

1. Borghese, F., and Saija, R. Electromagnetics and light scattering XIII. J Quant Spectrosc Radiat Transfer 2012;113:2277-2279.

2. Berg, MJ, and Videen, G. Concepts in electromagnetic scattering for particulatesystems characterization. J Quant Spectrosc Radiat Transfer 2013;131:1-2.

3. Purcell EM, Pennypacker CR. Scattering and absorption of light by nonspherical dielectric grains. Astrophys J 1973;186:705-14.

4. Penttilä A, Zubko E, Lumme K, Muinonen K, Yurkin MA, Draine B, Rahola J, Hoekstra AG, Shkuratov Yu. Comparison between discrete dipole implementations and exact techniques. J Quant Spectrosc Radiat Transfer 2007;106:417-36.

5. Zubko E, Petrov D, Grynko Y, Shkuratov Y, Okamoto H, Muinonen K, Nousiainen T, Kimura H, Yamamoto T, Videen G, 2010: Validity criteria of the discrete dipole approximation. Appl. Opt. 49, 1267-1279.

6. K. S. Yee, "Numerical solution of initial boundary value problems involving Maxwell's equations in isotropic media," IEEE Trans. Antennas Propagat. 14, 302307 (1966).

7. W. Sun, Q. Fu, and Z. Chen, "Finite-difference time-domain solution of light scattering by dielectric particles with a perfectly matched layer absorbing boundary condition," Appl. Opt. 38, 3141-3151 (1999).

8. Sun, WB, Videen, G, Fu, Q, Hu, YX, 2013: Scattered-field FDTD and PSTD algorithms with CPML absorbing boundary conditions for light scattering by aerosols. J. Quant. Spectrosc. Radiat. Transfer, 131, 166-174. 
9. Mishchenko, M. I., L. D. Travis, and D. W. Mackowski, 1996: T-matrix computations of light scattering by nonspherical particles: a review, J. Quant. Spectrosc. Radiat. Transfer 55, 535-575.

10. Petrov D, Shkuratov Y, Videen G, 2011: Electromagnetic wave scattering from particles of arbitrary shapes. J. Quant. Spectrosc. Radiative Transfer 112, 1636-1645.

11. Zubko E, Kimura H, Shkuratov Yu, Muinonen K, Yamamoto T, Okamoto H, Videen G. Effect of absorption on light scattering by agglomerated debris particles. J Geophys Res. 2012:117 D18205.

12. Zubko E, Muinonen K, Shkuratov Y, Videen G, Nousiainen T. Scattering of light by roughened Gaussian random particles. J Quant Spectrosc Radiat Transfer 2007;106:604-615.

13. Zubko, E, Muinonen, K, Munoz, O, Nousiainen, T, Shkuratov, Y, Sun, WB, Videen, G, 2013: Light scattering by feldspar particles: Comparison of model agglomerate debris particles with laboratory samples. J. Quant. Spectrosc. Radiat. Transfer, 131, 175-187145-152.

14. Mackowski D. The extension of Mie theory to multiple spheres. In: Hergert W, Wriedt Th, editors. The Mie theory. Berlin: Springer; 2012. p. 223-56.

15. Mackowski DW, Mishchenko MI. A multiple sphere T-matrix Fortran code for use on parallel computer clusters. J Quant Spectrosc Radiat Transfer 2011;112:2182-92.

16. Mishchenko MI, Liu L, Mackowski DW, “T-matrix modeling of linear depolarization by morphologically complex soot and soot-containing aerosols,” J Quant Spectrosc Radiat Transfer 2013;123:135-144.

17. Sun W, Z Liu, G Videen, Q Fu, K Muinonen, DM Winker, C Lukashin, Z Jin, B Lin, J Huang, 2012: For the depolarization of linearly polarized light by smoke particles. J. 
Quant. Spectrosc. Radiat. Transfer, 113, 233-237.

http://dx.doi.org/10.1016/j.jqsrt.2012.03.031.

18. Sun W, Hu Y, Lin B, Zhaoyan Liu Z., and Videen G, 2011: The impact of ice cloud particle microphysics on the uncertainty of ice water content retrievals. J. Quant. Spectrosc. Radiative Transfer 112, 189-196.

19. Zubko E, Muinonen K, Shkuratov Y, Videen G, 2013: Characteristics of cometary dust in the innermost coma derived from polarimetry by Giotto. MNRAS 430, 1118 1124.

20. Zubko E, Muinonen K, Shkuratov Y, Hadamcik E, Levasseur-Regourd AC, Videen G, 2012: Evaluating the carbon depletion found by the Stardust mission in Comet 81P/Wild 2, Astron. Astrophys. 544, L8.

21. Kolokolova L, Kimura H, Ziegler K, Mann I. Light-scattering properties of randomoriented aggregates: do they represent the properties of an ensemble of aggregates? J Quant Spectrosc Radiat Transfer 2006;100:199-206.

22. Rosenbush, V., Kiselev, N., Kolokolova, L., Velichko, S., Velichko, F., Antoniuk, K., Kolesnikov, S. (2009): Polarization properties of odd comet 17P/Holmes, J. Quant. Spectrosc. Radiat. Transfer, 110, 1719-1725.

23. Kolokolova, L., E. Petrova, H. Kimura, Effects of Interaction of Electromagnetic Waves in Complex Particles, In: Electromagnetic Waves (V. Zhurbenko, Ed.) , InTech, Vienna, ISSN 978-953-307-304-0, p. 173 - 202, 2011.

24. Lasue, J., Levasseur-Regourd, A. C. (2006): Porous irregular aggregates of submicron sized grains to reproduce cometary dust light scattering observations, J. Quant. Spectrosc. Radiat. Transfer, 100, 220-236. 
25. Petrova E.V., Tishkovets V.P., and Jockers, K., "Polarization of light scattered by Solar System bodies and the aggregate model of dust particles," Solar System Research 38 (4), 309-324 (2004).

26. Kimura, H., L. Kolokolova, and I. Mann, Optical properties of cometary dust: Constraints from numerical studies on light scattering by aggregate particles, Astron. Astrophys., 407, L5-L8, 2003.

27. Kimura, H., L. Kolokolova, and I. Mann, Light scattering by cometary dust numerically simulated with aggregate particles consisting of identical spheres, Astron. Astrophys., 449, 1243-1254, 2006.

28. I. Mann I., Kimura H. and Kolokolova L., A comprehensive model to describe light scattering properties of cometary dust, J. Quant. Spectrosc. Radiat. Transfer, 89, 291301 (2004).

29. Dlugach, J.M., M.I. Mishchenko, and D.W. Mackowski, "Scattering and absorption properties of polydisperse wavelength-sized particles covered with much smaller grains.” J. Quant. Spectrosc. Radiat. Transfer, 113, 2351-2355 (2012).

30. Tishkovets V.P. and Petrova E.V., "Coherent backscattering by discrete random media composed of clusters of spherical particles," J. Quant. Spectrosc. Radiat. Transfer, 127, 192-206 (2013).

31. Hadamcik, E., Levasseur-Regourd, A. C. (2003): Imaging polarimetry of cometary dust: different comets and phase angles, J. Quant. Spectrosc. Radiat. Transfer, 79-80, $661-678$.

32. Kolokolova L., and Kimura H., "Comet Dust as a Mixture of Aggregates and Solid Particles: Model Consistent with Ground-Based and Space-Mission Results," Earth, Planets and Space, 62, 17-22, 2010. 
33. E. Zubko, Y. Shkuratov, G. Videen, 2006: Discrete-dipole analysis of backscatter features of agglomerated debris particles comparable in size with wavelength. J. Quant. Spectrosc. Radiative Transfer 100, 483-488.

34. Muinonen K, Tyynelä J, Zubko E, Lindqvist H, Penttilä A, Videen G, 2011: Polarization of light backscattered by small particles. J. Quant. Spectrosc. Radiative Transfer, 112, 2193-2212.

35. Photopolarimetry in Remote Sensing, editors G. Videen, Y. Yatskiv, and M. Mishchenko (Kluwer, Dordrecht, 2004). ISBN: 1-4020-2366-9.

36. Petrova E.V., Tishkovets V.P., and Jockers, K., "Modeling of opposition effects with ensembles of clusters: Interplay of various scattering mechanisms," Icarus 188 (1), 233-245 (2007).

37. K. Muinonen, "Coherent backscattering of light by complex random media of spherical scatterers: Numerical solution," Waves in Random Media 14(3), 365-388 (2004).

38. Saxon, D. S., "Tensor Scattering Matrix for the Electromagnetic Field," Phys. Rev., 100, $1771(1955)$.

39. Muinonen, K. and Videen, G., “A Phenomenological single scatterer for studies of complex particulate media,” J. Quant. Spectrosc. Radiat. Transfer, 113, 2385 (2012).

40. Muinonen K, Mishchenko MI, Dlugach JM, Zubko E, Penttila A and Videen G, “Coherent backscattering verified numerically for a finite volume of spherical particles,” Astrophys. J. 760, 118 (2012).

41. Mishchenko, M. I., D. Goldstein, J. Chowdhary, and A. Lompado, 2013: Radiative transfer theory verified by controlled laboratory experiments. Opt. Lett. 38, 35223525 . 
42. S. Bondarenko, A. Ovcharenko, Y. Shkuratov, G. Videen, J. Eversole, and M. Hart, 2006: Light backscatter by surfaces composed of small spherical particles,” Appl. Opt. 45, 3871-3877.

43. Mishchenko, M. I., and L. Liu, 2009: Electromagnetic scattering by densely packed particulate ice at radar wavelengths: exact theoretical results and remote-sensing implications, Appl. Opt. 48, 2421-2426.

44. Y. Shkuratov, S. Bondarenko, A. Ovcharenko, C. Pieters, T. Hiroi, H. Volten, O. Muñoz and G. Videen, 2006: Comparative studies of the reflectance and degree of linear polarization of particulate surfaces and independently scattering particles. J. Quant. Spectrosc. Radiative Transfer 100, 340-358.

45. Yu. Shkuratov, S. Bondarenko, V. Kaydash, G. Videen, O. Muñoz and H. Volten, 2007: Photometry and polarimetry of particulate surfaces and aerosol particles over a wide range of phase angles. J. Quant. Spectrosc. Radiative Transfer 106, 487-508.

46. Yu. Shkuratov, A. Ovcharenko, E. Zubko, H. Volten, O. Muñoz, and G. Videen, 2004: The negative polarization of light scattered from particulate surfaces and of independently scattering particles, J. Quant. Spectrosc. Radiative Transfer 88, 267284. 

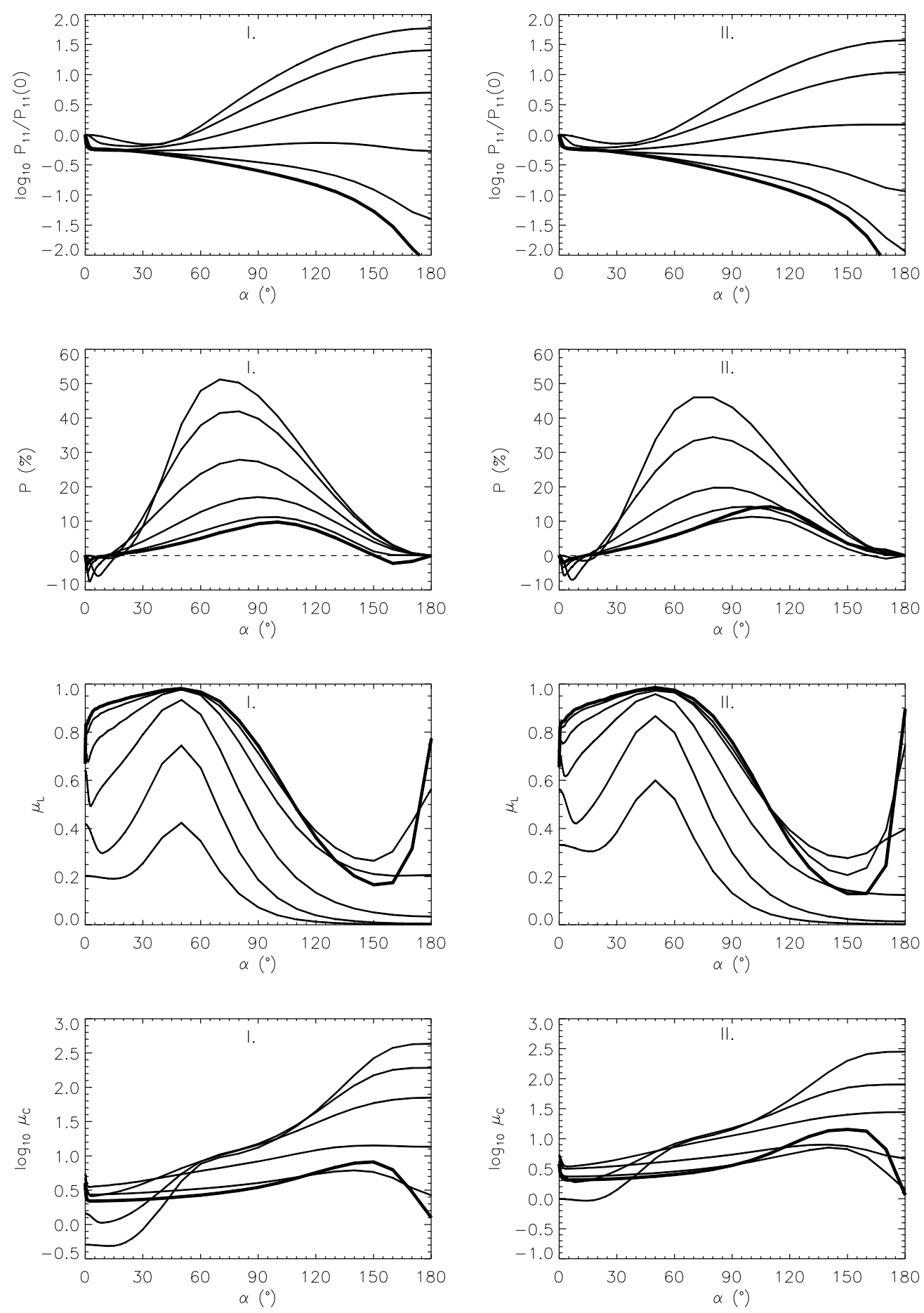

Figure 1: Light scattering by a spherical volume of particulate medium with a size parameter $k R=10,30,100,300,1000,3000(k R=3000$ is shown bold $)$ populated with spherical particles of size $k r=1.76$ and refractive index $m=1.50$. The first column is for agglomerates having densities $v=3 \%$ (I) and the second is for $v=6 \%$ (II). The separate rows show, from top to bottom, the phase-function intensity, linear polarization, linear polarization ratio, and circular polarization ratio. 

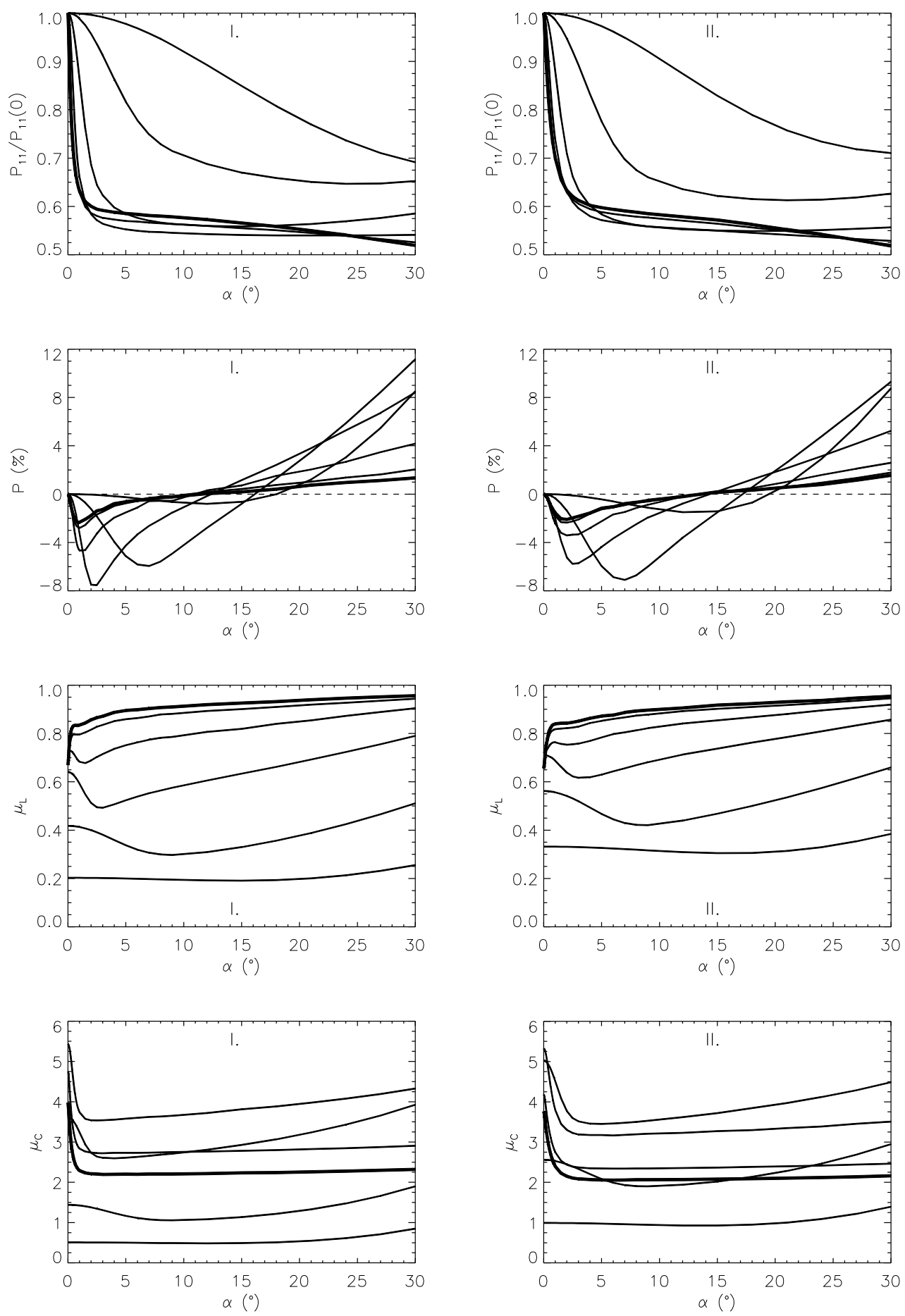

Figure 2: As in Fig. 1, but for the backscattering region. 



Figure 3: Plots of scattering parameters as a function of agglomerate size $k R$. Plots show magnitudes of a) $P_{\min }$, b) $P_{\max }$, phase angle positions of c) $P_{\min }$, d) $P_{\max }$, cross section of e) linear polarization $\mu_{\mathrm{L}}$ and circular polarization $\mu_{\mathrm{C}}$ at $\alpha=0^{\circ}$. Closed circles are for $v=3.0 \%$, and open circles are for $v=6.0 \%$. 